\title{
Análise do conteúdo de Evolução em livros didáticos do Ensino Fundamental brasileiro
}

\author{
João Vitor Tomotani ${ }^{1}$ \& Rodrigo Brincalepe Salvador ${ }^{2,3}$
}

(1) Universidade de São Paulo, Escola Politécnica, Departamento de Engenharia de Produção, Av. Prof. Almeida Prado 531, Cidade Universitária 05508-900, São Paulo, Brasil. E-mail: t.jvitor@gmail.com

(2) Staatliches Museum für Naturkunde Stuttgart, Stuttgart, Germany.

(3) Eberhard Karls Universität Tübingen, Tübingen, Germany. E-mail: salvador.rodrigo.b@gmail.com

Tomotani J.V. \& Salvador R.B. (2017) Análise do conteúdo de Evolução em livros didáticos do Ensino Fundamental brasileiro. Pesquisa e Ensino em Ciências Exatas e da Natureza, 1(1): 05-18.

Resumo: A Teoria da Evolução é um dos conceitos científicos mais importantes, possuindo caráter central na Biologia como teoria unificadora. Entretanto, verifica-se que, de modo geral, o tema tem sido negligenciado ou mal apresentado ao público, sendo que atualmente grande parte da população, em todos os níveis de formação, não compreende ou mesmo não acredita na Evolução. Este trabalho teve como objetivo analisar livros didáticos aprovados pelo Programa Nacional do Livro Didático (PNLD) e utilizados no Ensino Fundamental II, visando identificar erros conceituais, questões de linguagem, a presença do "Lamarckismo" e do Criacionismo, que possam se constituir em obstáculos no ensino da Evolução. Os autores reconheceram diversos problemas nos livros didáticos capazes de gerar confusão conceitual em estudantes; dentre estes, o mais problemático é não tratar a Evolução como tema central da Biologia. Ainda assim, verifica-se que é possível expor o tema de forma coerente, sem necessariamente recorrer a simplificações que gerem erros conceituais.

Palavras chave: Criacionismo, Lamarckismo, Darwinismo, scala naturae, Ensino de Ciências, PNLD.

\section{Content analysis of the subject of evolution in text books used in elementary schools in Brazil}

Abstract: The Theory of Evolution is not only the unifying force in Biology, but also one of the most important scientific concepts ever developed. However, the topic has always been neglected or misrepresented, resulting in a population that do not understand (or outright do not believe in) Evolution. The present work consists in a content analysis of Evolution in Brazilian Middle School textbooks. Its aims included the search for obstacles to the understanding of Evolution, such as: conceptual errors, language problems, "Lamarckism" and Creationism. We conclude that many of these obstacles are indeed present in textbooks, hampering the students' grasp on Evolution; among these, the gravest is not representing Evolution as Biology's central theory. Nevertheless, we also observed that it is indeed possible to develop the theme in a coherent manner, without recourse to over-simplifications ("dumbing down") which may result in conceptual mistakes.

Key words: Creationism, Lamarckism, Darwinism, scala naturae, Science education, PNLD.

\section{Introdução}

A Teoria da Evolução reconhecidamente consiste em um dos mais importantes conceitos científicos já estudados, capaz de atuar como um guia para a unificação das ciências biológicas. Um relatório desenvolvido no Reino Unido em 1998 buscou reavaliar o ensino das ciências nas escolas, visando uma educação compatível com as habilidades e tecnologias necessárias para um currículo além do ano 2000. Tal relatório considerou o ensino da Teoria da Evolução como essencial, classificando-o como "um dos mais poderosos e significantes conhecimentos que possuímos” (Millar \& Osborne 1998: 13). Por conta da relevância da 
compreensão da Evolução no estudo da Biologia, tornou-se célebre a frase "nada em biologia faz sentido exceto à luz da evolução" (Dobzhansky 1973), ainda que frequentemente citada fora de seu contexto original. Por conta desses fatores, seria natural que a Evolução fosse um conceito central em qualquer currículo de ciências (Hokayem \& Boujaoude 2008). Entretanto, a Evolução tem um histórico de ser negligenciada ou mal representada em livros didáticos e na mídia em geral (Skoog 1979; Gould 1983a). Além disso, o tema raramente é apresentado ao público como o conceito central da Biologia (Jeffery \& Roach 1994; Skoog \& Bilica 2002). Tais fatores se tornam ainda mais preocupantes quando se verifica a quantidade de trabalhos que acusam a não compreensão da Teoria da Evolução ou mesmo a descrença em sua existência, por estudantes (e professores), em todos os níveis de formação (Allmon 2011; Almeida 2012).

Adicionalmente, outra barreira se coloca à frente do ensino de Evolução: o Criacionismo, onde supõe-se que os organismos foram criados ex nihilo por um agente sobrenatural. Em uma pesquisa realizada pelo IBOPE (IBOPE 2004), identificou-se que $31 \%$ da população brasileira acredita que o ser humano surgiu nos últimos 10 mil anos, da forma como é hoje; 54\% acredita que houve um processo de evolução ao longo de milhões de anos, sendo este processo dirigido por Deus; e somente 9\% acredita que o processo de evolução se deu de forma aleatória, sem o envolvimento de uma figura divina. Números semelhantes foram identificados em outros países, sendo observada uma forte correlação entre o descrédito na Teoria da Evolução e fatores como a religião cristã, o nível de educação formal da população e o conservadorismo político (Mazur 2005; Miller 2006; Penteado et al. 2012). A mesma pesquisa do IBOPE apontou que $89 \%$ dos brasileiros entrevistados defende que o Criacionismo seja ensinado na escola, sendo que $75 \%$ acredita que o Criacionismo deva ser ensinado no lugar do Evolucionismo. Conflito semelhante foi encontrado na pesquisa de Oliveira \& Bizzo (2011), a qual identificou que níveis de aceitação dos tópicos referentes à evolução biológica por parte dos alunos são influenciados por sua religião.

Verifica-se, assim, que distorções no ensino da Evolução são ainda mais problemáticas do que seriam em outros tópicos do ensino em ciências, não só por se tratar de um tema polêmico e delicado, mas também (e especialmente) por ter um caráter central na Biologia como teoria unificadora (Moody 1996; Almeida \& Falcão 2010).

Poucos estudos trataram da abordagem desse tema nas escolas e nos livros didáticos brasileiros. Bizzo (1994) verificou que os alunos frequentemente confundem conceitos de uso e desuso com Seleção Natural. Almeida (2012) verificou uma forte presença da concepção criacionista entre os alunos, particularmente na questão do surgimento da espécie humana, identificando como principais obstáculos a dificuldade em aceitar o "acaso" como um importante mecanismo evolutivo, e a tendência dos alunos em obrigatoriamente relacionar Evolução com ateísmo. Borges \& Lima (2007) argumentaram que a Evolução ocupa o último lugar na lista de temas explorados no ensino de Biologia, algo totalmente oposto ao caráter fundamental do tema. Todos esses fatores fazem com que o ensino de Evolução seja uma área que necessita de ampla e constante atenção (Good 1992; Smith et al. 1995).

\section{Importância dos livros didáticos no ensino}

Os livros didáticos têm um papel central no ensino, determinando amplamente o que é ou não estudado (Skoog 1979; Swarts et al. 1994). Segundo Martins (2006), o livro didático possui importância indiscutível no cenário da educação no Brasil, tendo forte relação com as práticas constitutivas da escola e do ensino escolar. Tal importância é confirmada, dentre outros fatores, "pelo debate em torno da sua função na democratização de saberes socialmente legitimados e relacionados a diferentes campos de conhecimento, pela polêmica acerca do seu papel como estruturador da atividade docente, pelos interesses econômicos em torno da sua produção e comercialização, e pelos investimentos de governos em programas de avaliação" (Martins 2006: 118).

Os livros didáticos podem desempenhar um papel ainda mais central como autoridades em tópicos polêmicos, como a Evolução, fazendo com que os professores recorram a eles como 
forma de assegurar a legitimidade daquilo que estão ensinando (e.g., Zimmerman 1987; Goldston \& Kyzer 2009). Como colocado por Goldston \& Kyzer (2009: 779): “Teachers who chose to teach evolution, depended on the authority of the textbook to legitimize the topic and provide safety from potential consequences."

Por conta da importância dos livros didáticos no processo de ensino, algumas críticas em relação a esses se mostram relevantes. Amaral \& Megid Neto (1997) e Megid Neto \& Fracalanza (2003) questionaram a qualidade dos livros, ao argumentar que as editoras são muito motivadas por critérios mercadológicos, como aspectos gráficos editoriais e perspectiva de uso dos livros em períodos maiores que um ano, em detrimento da qualidade do conteúdo.

Os livros didáticos de Biologia do Ensino Médio brasileiro também foram amplamente criticados por Bizzo (1994), o qual argumentou que parte dos conteúdos sobre Evolução havia sido claramente baseada em publicações para o público geral, contendo inúmeras instâncias de descuidado na linguagem que poderiam resultar em compreensão errônea pelo leitor. Entretanto, o tópico foi escassamente analisado em livros brasileiros nas duas décadas seguintes. Uma análise referente ao Ensino Médio foi conduzida recentemente por Dalapicolla et al. (2015), os quais chegaram à conclusão de que a abordagem da Evolução nos livros didáticos é superficial e fragmentada. Segundo Dalapicolla et al. (2015), a forma descritiva utilizada para a exposição dos conceitos evolutivos não auxilia na construção de uma visão evolutiva integrada. Esses autores ainda apontaram que há diferenças significativas nas abordagens e qualidade da exposição do conteúdo de Evolução entre as coleções, ainda que todas tenham sido aprovadas pelo Programa Nacional do Livro Didático (PNLD).

O ensino de Evolução tem maior ênfase no Ensino Médio e são os livros didáticos desse estágio que geralmente são analisados (e.g., Skoog 1979, 1984, 2005; Rosenthal 1985; Bizzo 1994; Jiménez-Aleixandre 1994). Entretanto, o ensino da Evolução durante as etapas iniciais da formação do aluno (i.e., no Ensino Fundamental II) é, no mínimo, igualmente importante (Jeffery \& Roach 1994; Department for Education 2013). Contudo, os livros didáticos do Ensino Fundamental raramente recebem a atenção das análises da mesma forma como são realizadas com livros do Ensino Médio (Swarts et al. 1994).

Assim, dado(a) (1) a posição fundamental que a Evolução tem na Biologia, (2) a má apresentação ou total negligência do tema, (3) a importância de ensinar os conceitos desde cedo e (4) o papel central que os livros didáticos têm no ensino, o presente trabalho teve como objetivo analisar como a Evolução Biológica é apresentada nos livros didáticos nacionais do Ensino Fundamental II.

\section{Material e Métodos}

Para a realização do presente trabalho, sete coleções de livros didáticos do Ensino Fundamental II foram selecionadas, contando ao todo com nove livros, dado que em duas coleções o tema Evolução encontrou-se separado em dois volumes. Os livros utilizados foram elaborados pelos seguintes autores: Bröckelmann (2008), Canto (2009a, 2009b), Godoy \& Ogo (2012), Santana (2012), Santana \& Mozena (2012), Stern (2009), Trivellato et al. (2008) e Usberco et al. (2012). Todos os livros aqui empregados pertencem ao $7^{\circ}$ ano (antiga $6^{\mathrm{a}}$ série), exceto os dois livros adicionais supracitados, que pertencem ao $9^{\circ}$ ano (antiga $8^{\mathrm{a}}$ série). As coleções analisadas são as listadas pelo PNLD 2014 (BRASIL 2013), contando ao todo com 20 coleções de Ciências. Todos os livros analisados são as versões do PNLD. A escolha do material do PNLD se dá pelo fato de que a maioria das escolas brasileiras usa tais livros: a totalidade das escolas públicas, naturalmente, mas também boa parte das escolas particulares, que se guiam pelas coleções selecionadas pelo PNLD ao adotar o material didático.

A metodologia utilizada é uma adaptação da "análise de conteúdo" (“content analysis") (e.g., Rist 1982; Krippendorff 2013) comumente usada em livros didáticos, estando baseada na literatura internacional da área.

A princípio, o tema "Evolução" foi subdividido, a priori, em categorias (tópicos) para facilitar a comparação entre as obras. Essa abordagem foi usada extensivamente por Skoog 
(1979, 1984), Rosenthal (1985) e Swarts et al. (1994) para livros de Biologia do Ensino Médio. As categorias escolhidas na presente análise foram bastante semelhantes às adotadas por Skoog (1979, 1984), mas simplificadas para a realidade do Ensino Fundamental. Como o alvo do presente trabalho são livros do Ensino Fundamental II, que contém uma forma mais simplificada da matéria, diminuímos o número de categorias usadas, de modo semelhante à metodologia de Jeffery \& Roach (1994). Cada categoria tem uma quantidade de temas que caem em seu domínio, sendo totalmente excludentes umas das outras. As categorias (e os temas que contém) são: (1) Ciência (definição, funcionamento e método científico); (2) Evolução (definição, histórico, Darwin, Wallace e Lamarck); (3) Criacionismo (doutrinas e mitos); (4) Mecanismos (variabilidade, hereditariedade, adaptação, Seleção Natural, especiação, isolamento geográfico/reprodutivo e interações ecológicas); (5) Evidências (Seleção Artificial, fósseis, caracteres morfoanatômicos, fisiologia, embriologia e biologia molecular); (6) Sistemática (conceito de espécie, taxonomia, filogenia, cladogramas e ancestral comum); (7) Geologia/Paleontologia (idade da Terra, tempo geológico, tectônica de placas, deriva continental, biogeografia e extinções); (8) Origem da vida (experimentos, formação de moléculas complexas, células e micro-organismos); (9) Origem e evolução das plantas e animais (origem e diversificação dos grandes grupos, invasão do ambiente terrestre, polinização e evolução humana). Dentro desta última categoria, destaque especial foi dado para uma única subcategoria: evolução dos primatas e humanos.

Os trabalhos anteriores usavam o método de contar o número de palavras dedicadas a cada categoria. Entretanto, tal método apenas lidava com o texto em si (Levin \& Lindbeck 1979; Skoog 1979, 1984; Rosenthal 1985), deixando de fora itens como ilustrações e exercícios, igualmente importantes para o aprendizado, mas raramente analisados (Swarts et al. 1994; Catley et al. 2010). Assim, no presente trabalho, faz-se o uso da contagem de páginas (em incrementos de $1 / 4$ de página) para incluir esses outros itens. Cada parágrafo, ilustração e exercício dos livros foram analisados e alocados em uma das categorias, resultando na proporção em que cada categoria ocupa em cada livro.

Entretanto, somente a proporção de um tema no texto não indica como ela é apresentada e ensinada, tampouco reflete a importância que os autores dão ao tema (Krippendorff 2013). Trabalhos anteriores apenas definiram essa proporção ou tentaram análises vagas, de forma não sistemática, do conteúdo dos textos (e.g., Moody 1996). Assim sendo, no presente trabalho, além de apresentarmos a proporção dos livros dedicados a cada tema, também oferecemos uma forma mais sistemática de analisar a qualidade do conteúdo, como descrito abaixo.

Cada uma das nove categorias supracitadas foi analisada e classificada em graus de erro do seguinte modo (exceção: categoria "Criacionismo", que não pode ser julgada dessa forma): (0) Categoria ausente; (1) Erro Crítico (teoria errada, informações e/ou escrita tendenciosa referente à religião e/ou política); (2) Erro Grave (erros gritantes, informações falsas, exemplos conflitantes com teoria); (3) Erro Médio (informações erradas, problemáticas e prejudiciais ao aprendizado, informações críticas ausentes, exemplos inapropriados); (4) Erro Mínimo (informação errada de pouca monta, exemplos incompletos, má escolha de palavras); (5) Correto. Assim sendo, é possível avaliar não só erros conceituais, mas falhas em se apresentar o conteúdo ao aluno (Martins 2006). Isso se mostra importante, principalmente por causa da linguagem utilizada; o tema de Evolução, tanto no ensino quanto na mídia, é saturado de linguajar e ideias errôneas (Bizzo 1994), principalmente referentes à scala naturae e às expressões "Lamarckistas".

Finalmente, analisamos outros pontos adicionais. Em primeiro lugar, checamos o número de vezes que a palavra "evolução" ocorre nos títulos e subtítulos dos capítulos, nos glossários e em índices remissivos (Skoog 1979, 1984). Em segundo lugar, checamos se havia algum tipo de "retratação" (“disclaimer") no prefácio ou introdução dos livros "se desculpando" pela Evolução, semelhante ao relatado por Rosenthal (1985) em livros norte-americanos. 


\section{Resultados}

Os resultados principais da análise de conteúdo são apresentados na Tabela 1. Tal tabela mostra a proporção e o "grau de erro" presente em cada categoria nos respectivos livros. A Tabela 2 traz informações adicionais importantes para a análise dos quesitos mais específicos que poderiam ficar "escondidos" dentro dos dados da análise de conteúdo. Por exemplo, há informações sobre quais desses quesitos são abordados nos livros.

Um ponto positivo dos livros analisados é que nenhum deles apresentou o disclaimer se desculpando pela evolução (algo comum nos EUA) (Rosenthal 1985). Entretanto, alguns livros (e.g., Godoy \& Ogo 2012; Santana 2012; Santana \& Mozena 2012; Usberco et al. 2012) ainda incluem o Criacionismo antes de explicar Evolução.

Charles Darwin foi sempre citado nos textos, mas somente na metade dos casos (Bröckelmann 2008; Sterm 2009; Godoy \& Ogo 2012; Santana \& Mozena 2012; Usberco et al. 2012) Alfred R. Wallace foi mencionado. Lamarck, entretanto, aparece em todas as coleções (exceto em Trivellato et al. 2008) e isso pode ser um problema, como discutido na próxima seção.

Um tópico essencial para o entendimento da Seleção Natural (e, portanto, da Evolução em si) é a Seleção Artificial. Infelizmente, esse tópico raramente é abordado ou apontado como uma das evidências que Darwin usou para a Seleção Natural. Os livros que trazem o tema são: Bröckelmann (2008), Trivellato et al. (2008), Canto (2009b) e Santana \& Mozena (2012). Contudo, tais livros raramente exploram o tema o suficiente ou o colocam como essencial. Seleção Sexual, outro tópico importante, não apareceu em nenhum livro.

Em geral, a categoria do que é Ciência (como ela funciona, o método científico etc.) raramente foi abordada. Ao analisar todos os livros das coleções, procurou-se esta categoria, mas sem sucesso. Quando abordada nos livros, tal categoria foi discorrida de forma fragmentada e não muito clara. Deste modo, é extremamente provável que o aluno continue sem saber o que é Ciência.

Abaixo, apresentamos uma visão geral de cada livro (os livros da mesma coleção são analisados em conjunto), com informações adicionais às mencionadas logo acima. Deve-se notar que esta não é uma resenha sobre os livros ou indicação de qual é melhor ou pior e qual deve ser adotado ou evitado. Simplesmente, analisou-se um tema (Evolução) dentro dos quatro livros que compõe cada coleção de Ciências do Ensino Fundamental II.

Bröckelmann (2008): O livro traz explicações bem superficiais sobre todos os temas relacionados à Evolução. A tentativa do autor de resumir os conteúdos e deixá-los mais fáceis, deixam-nos na verdade com alguns erros conceituais. Assim, o seu maior problema é a falta de conteúdo e de conexão lógica entre os temas.

Canto (2009a, 2009b): O primeiro livro (7 ${ }^{\circ}$ ano; Canto 2009a) conta com um ótimo encadeamento lógico na sequência dos temas (apesar do conteúdo referente aos fósseis, importante para diversos pontos, ser encontrado somente ao final do livro). Além disso, o livro conta com pouco espaço dedicado a Lamarck, o que é um bom sinal, e presta muita atenção para evitar vícios de fala "Lamarckista" e o vocabulário típico da scala naturae. No segundo livro (90 ano; Canto 2009b), o autor retoma os temas de Evolução e Seleção Natural com base no novo conteúdo de Genética (hereditariedade, variação etc.). Entretanto, ao simplificar demais o assunto, o autor cometeu alguns erros conceituais.

Godoy \& Ogo (2012): Um dos livros mais completos em conteúdo, apesar de ainda faltar alguns conceitos-chave (e.g., Seleção Artificial). A apresentação dos filos está organizada no típico modo scala naturae, com os primatas e o homem como sendo os últimos na cadeia dos seres; no entanto, o texto evita completamente o vocabulário típico da scala naturae. A abordagem mais relevante aqui reconhecida foi o livro contar com explicações sobre origens, evolução e relações internas em plantas e vertebrados (o tópico é raramente abordado nos outros livros aqui analisados). $\mathrm{O}$ aspecto mais problemático deste livro foi a presença do Criacionismo, apesar do assunto ter sido abordado somente como uma lenda do Vietnã. Juntamente com Usberco et al. (2012), é o livro com o menor número de erros e problemas (Tabela 1). 
Tabela 1. Resultado da análise de conteúdo de Evolução em livros didáticos nacionais com base em nove categorias (e uma subcategoria: evolução em primatas e humanos). As colunas indicam o número total e a proporção de páginas dedicadas a cada categoria em cada livro, assim como o "grau de erro" do conteúdo. $\mathrm{pg}$ = página $(\mathrm{s})$.

\begin{tabular}{|c|c|c|c|c|c|c|c|c|c|}
\hline & \multicolumn{3}{|c|}{ Bröckelmann (2008) } & \multicolumn{3}{|c|}{ Canto (2009a) } & \multicolumn{3}{|c|}{ Canto (2009b) } \\
\hline CATEGORIAS & $\mathrm{n}^{0} \mathrm{pg}$ & $\% \mathrm{pg}$ & Erro & $\mathrm{n}^{0} \mathrm{pg}$ & $\% \mathrm{pg}$ & Erro & $\mathrm{n}^{\circ} \mathrm{pg}$ & $\% \mathrm{pg}$ & Erro \\
\hline Ciência & - & - & 0 & - & - & 0 & 0.5 & 0.18 & 3.0 \\
\hline Evolução & 6.5 & 2.36 & 3.0 & 3.75 & 1.58 & 5.0 & 2.75 & 0.97 & 4.0 \\
\hline Criacionismo & - & - & - & - & - & - & - & - & - \\
\hline Mecanismos & 2.5 & 0.91 & 4.0 & 12.5 & 5.27 & 5.0 & 2.5 & 0.88 & 4.0 \\
\hline Evidências & 2.0 & 0.72 & 4.0 & 6.0 & 2.53 & 4.0 & 2.5 & 0.88 & 3.0 \\
\hline Sistemática & 12.25 & 4.44 & 4.0 & 20.5 & 8.65 & 4.0 & - & - & 0 \\
\hline Geologia/Paleontologia & - & - & 0 & 6.0 & 2.53 & 5.0 & - & - & 0 \\
\hline Origem da vida & 5.0 & 1.81 & 5.0 & - & - & 0 & 1.0 & 0.35 & 4.0 \\
\hline $\begin{array}{l}\text { Origem e evolução das } \\
\text { plantas e animais }\end{array}$ & 4.5 & 1.63 & 4. 0 & 5.0 & 2.11 & 4.0 & - & - & 0 \\
\hline \multirow[t]{2}{*}{ Primatas/humanos } & 1.5 & 0.54 & 4.0 & 1.75 & 0.74 & 4.0 & - & - & 0 \\
\hline & \multicolumn{3}{|c|}{ Godoy \& Ogo (2012) } & \multicolumn{3}{|c|}{ Santana (2012) } & \multicolumn{3}{|c|}{ Santana \& Mozena (2012) } \\
\hline CATEGORIAS & $\mathrm{n}^{0} \mathrm{pg}$ & $\% \mathrm{pg}$ & Erro & $\mathrm{n}^{0} \mathrm{pg}$ & $\% \mathrm{pg}$ & Erro & $\mathrm{n}^{0} \mathrm{pg}$ & $\% \mathrm{pg}$ & Erro \\
\hline Ciência & 3.5 & 1.14 & 4.0 & - & - & 0 & - & - & 0 \\
\hline Evolução & 9.0 & 2.94 & 4.0 & - & - & 0 & 6.25 & 1.93 & 4.0 \\
\hline Criacionismo & 0.75 & 0.25 & - & - & - & - & 2.0 & 0.62 & - \\
\hline Mecanismos & 3.5 & 1.14 & 4.0 & - & - & 0 & 4.25 & 1.31 & 4.0 \\
\hline Evidências & 1.0 & 0.33 & 4.0 & - & - & 0 & 5.0 & 1.54 & 4.0 \\
\hline Sistemática & 14.75 & 4.82 & 4.0 & 23 & 7.49 & 2.0 & 2.75 & 0.85 & 5.0 \\
\hline Geologia/Paleontologia & 3.0 & 0.98 & 5.0 & - & - & 0 & 4.75 & 1.47 & 2.0 \\
\hline Origem da vida & 4.75 & 1.55 & 5.0 & - & - & 0 & 5.5 & 1.70 & 3.0 \\
\hline $\begin{array}{l}\text { Origem e evolução das } \\
\text { plantas e animais }\end{array}$ & 9.75 & 3.19 & 4.0 & - & - & 0 & 11 & 3.40 & 3.0 \\
\hline \multirow[t]{2}{*}{ Primatas/humanos } & - & - & 0 & - & - & 0 & 6.75 & 2.08 & 4.0 \\
\hline & \multicolumn{3}{|c|}{ Stern (2009) } & \multicolumn{3}{|c|}{ Trivellato et al. (2008) } & \multicolumn{3}{|c|}{ Usberco et al. (2012) } \\
\hline CATEGORIAS & $\mathrm{n}^{\mathbf{0}} \mathrm{pg}$ & $\% \mathrm{pg}$ & Erro & $\mathrm{n}^{0} \mathrm{pg}$ & $\% \mathrm{pg}$ & Erro & $\mathrm{n}^{\mathbf{0}} \mathrm{pg}$ & $\% \mathrm{pg}$ & Erro \\
\hline Ciência & - & - & 0 & - & - & 0 & 0.25 & 0.09 & 5.0 \\
\hline Evolução & 4.75 & 1.61 & 3.0 & 1.75 & 0.75 & 2.0 & 8.5 & 3.07 & 5.0 \\
\hline Criacionismo & - & - & - & - & - & - & 0.5 & 0.18 & - \\
\hline Mecanismos & 3.25 & 1.10 & 4.0 & 5.0 & 2.14 & 3.0 & 0.25 & 0.09 & 5.0 \\
\hline Evidências & 2.5 & 0.85 & 4.0 & 3.5 & 1.50 & 4.0 & 2.25 & 0.81 & 5.0 \\
\hline Sistemática & 14 & 4.75 & 3.0 & 8.0 & 3.42 & 4.0 & 13.5 & 4.87 & 4.0 \\
\hline Geologia/Paleontologia & 2.0 & 0.68 & 5.0 & 2.0 & 0.85 & 3.0 & 1.75 & 0.63 & 5.0 \\
\hline Origem da vida & 7.25 & 2.46 & 5.0 & - & - & 0 & 7.0 & 2.53 & 5.0 \\
\hline $\begin{array}{l}\text { Origem e evolução das } \\
\text { plantas e animais }\end{array}$ & 7.75 & 2.63 & 3.0 & 10.25 & 4.38 & 3.0 & 13.25 & 4.78 & 4.0 \\
\hline Primatas/humanos & 0.5 & 0.17 & 4.0 & - & - & 0 & 4.25 & 1.53 & 5.0 \\
\hline
\end{tabular}

Santana (2012) e Santana \& Mozena (2012): Essa coleção é interessante por trazer o conteúdo separado não por disciplinas (e.g., Biologia, Química, Física e Geologia), mas por grandes temas. Entretanto, tal abordagem deixa de fora diversos tópicos importantes, além de fazer com que muitos conteúdos que seriam interligados percam a sua conexão lógica, por serem abordados em anos diferentes e sem uma referência clara ao conteúdo aprendido nos anos anteriores. No primeiro livro ( $7^{\circ}$ ano; Santana 2012), o único tópico presente é Sistemática, o qual foi abordado de maneira muito simplificada. Isto fez com que a autora cometesse diversos erros graves em seu texto. Além disso, o capítulo começou com um exemplo inapropriado e tendencioso, a lenda da "Arca de Noé". Finalmente, a palavra "evolução" sequer aparece no livro.

O segundo livro ( $9^{\circ}$ ano; Santana \& Mozena 2012) tem uma apresentação bastante confusa dos tópicos, o que dificulta a absorção do conteúdo. As autoras usaram termos típicos da scala naturae (i.e., "espécie mais evoluída de todas", ao comparar os vertebrados aos 
invertebrados). Curiosamente, as autoras tiveram cuidado com isso na parte sobre evolução humana, falando que não foi um evento linear e que não havia uma espécie "mais evoluída". Um ponto interessante, que também quebra a ideia de scala naturae, foi a inclusão de um cladograma envolvendo vertebrados, no qual os mamíferos aparecem em uma posição intermediária (antes das tartarugas, como de praxe) e as aves no final. Juntamente com Trivellato et al. (2008), é uma das coleções estudadas em que encontramos "erros graves", de acordo com a nossa classificação.

Tabela 2. Quesitos adicionais de importância para a análise de conteúdo, como presença de abordagem de certos tópicos específicos.

\begin{tabular}{|c|c|c|c|c|c|}
\hline QUESITO & $\begin{array}{l}\text { Bröckelmann } \\
\text { (2008) }\end{array}$ & $\begin{array}{l}\text { Canto } \\
\text { (2009a) }\end{array}$ & $\begin{array}{l}\text { Canto } \\
(2009 b)\end{array}$ & $\begin{array}{l}\text { Godoy \& Ogo } \\
\text { (2012) }\end{array}$ & $\begin{array}{c}\text { Santana } \\
(2012)\end{array}$ \\
\hline Total de páginas do livro* & 276 & 237 & 284 & 306 & 307 \\
\hline $\begin{array}{l}\text { Ocorrência da palavra } \\
\text { "evolução" em títulos }\end{array}$ & 4 & 5 & 3 & 2 & 0 \\
\hline Presença de Criacionismo & $\mathrm{N}$ & $\mathrm{N}$ & $\mathrm{N}$ & $\mathrm{S}$ & S \\
\hline Presença de Lamarck & S & S & S & S & $\mathrm{N}$ \\
\hline Presença de Wallace & S & $\mathrm{N}$ & $\mathrm{N}$ & S & $\mathrm{N}$ \\
\hline $\begin{array}{l}\text { Presença de seleção } \\
\text { artificial }\end{array}$ & S & $\mathrm{N}$ & S & $\mathrm{N}$ & $\mathrm{N}$ \\
\hline QUESITO & $\begin{array}{c}\text { Santana \& Mo } \\
(2012)\end{array}$ & ena & $\begin{array}{l}\text { Stern } \\
(2009)\end{array}$ & $\begin{array}{l}\text { Trivellato et al. } \\
\quad(2008)\end{array}$ & $\begin{array}{l}\text { Usberco et al. } \\
\quad(2012)\end{array}$ \\
\hline Total de páginas do livro* & 324 & & 295 & 234 & 277 \\
\hline $\begin{array}{l}\text { Ocorrência da palavra } \\
\text { "evolução" em títulos }\end{array}$ & 7 & & 4 & 3 & 3 \\
\hline Presença de Criacionismo & S & & $\mathrm{N}$ & $\mathrm{N}$ & $S$ \\
\hline Presença de Lamarck & $S$ & & $S$ & $\mathrm{~N}$ & S \\
\hline Presença de Wallace & S & & S & $\mathrm{N}$ & S \\
\hline $\begin{array}{l}\text { Presença de seleção } \\
\text { artificial }\end{array}$ & $S$ & & $\mathrm{~N}$ & S & $\mathrm{N}$ \\
\hline
\end{tabular}

*O número total de páginas conta somente com o conteúdo do livro em si, excluindo dados bibliográficos, índices e bibliografia.

Stern (2009): Apesar de apresentar diversos cladogramas ao longo do texto, o autor manteve a ordenação e o vocabulário típicos da scala naturae (i.e., "mais primitivo" à esquerda; macacos são espécies "menos desenvolvidas" que o homem etc.). Explicações sobre origem, evolução e relações internas nos grupos só foram apresentadas para as plantas.

Trivellato et al. (2008): Este livro apresentou uma série de problemas. Os tópicos avaliados não demonstraram uma explicação mais aprofundada e os conceitos não foram organizados de forma a estabelecer uma conexão lógica. Além disso, diversos tópicos importantes (como especiação e hereditariedade) não foram mencionados. Evolução é o último capítulo do livro e o volume todo apresentou vocabulário da scala naturae (i.e., "mais primitivo", "menos desenvolvido"). Explicações sobre origem, evolução e relações internas nos grupos só foram apresentadas para as plantas. Juntamente com Santana (2012) e Santana \& Mozena (2012), é uma das coleções estudadas em que encontramos "erros graves", de acordo com a nossa classificação explicitada acima.

Usberco et al. (2012): É o livro mais completo em termos de conteúdo de Evolução; o encadeamento lógico dos temas e as explicações são as mais claras dentre todas as coleções. Embora a apresentação dos grupos esteja organizada no modo típico da scala naturae, com os primatas (e, portanto) o homem como os últimos apresentados, houve o cuidado de evitar o vocabulário da scala naturae. Além disso, houve cuidado especial no vocabulário para definir termos, apontando e explicando erros comuns na fala e na imprensa (como "o ser humano descende do macaco"). De especial atenção são os seguintes itens presentes no livro: um cladograma dos vertebrados, no qual os mamíferos encontram-se em posição intermediária (antes das tartarugas), com as aves no final; uma abordagem sobre a origem e relações 
filogenéticas de todos os cordados, moluscos, artrópodes e fungos (mas não de plantas); a abundância de informações e evidências fósseis. Um ponto baixo é a inclusão do Criacionismo bíblico. Juntamente com Godoy \& Ogo (2012), é um dos livros estudados com o menor número de erros e problemas.

\section{Discussão}

Como argumentado por Jiménez-Aleixandre (1994), o principal objetivo do ensino de Evolução é permitir que os alunos compreendam a ideia de Seleção Natural, de modo que consigam aplicá-la para interpretar qualquer fenômeno biológico. Logicamente, isto faz sentido para todas as ciências, como explicitado desde o final do século XIX: "the true teaching of Science consists, not merely in imparting the facts of Science, but in habituating the pupil to observe... to reason... and to check... by further observation and experiment" (Devonshire Commission 1875 [Sixth Report] apud Maclure 1986: 108). Ou ainda, como colocado de modo mais abrangente por Freire (1996), ensinar não é só transmitir conhecimentos, e sim criar possibilidades de autonomia.

Na sessão anterior, ressaltamos os tópicos mais problemáticos que foram encontrados na análise de conteúdo dos livros didáticos. Abaixo, esses tópicos são discutidos em mais detalhes e em um contexto mais amplo.

\section{A questão do lugar e do tempo}

Era algo comum, até poucas décadas atrás, que a parte do livro didático dedicada à Evolução fosse a última (Gould 1983b; Skoog 1984). Tal posição no livro já é em si absurda, tendo em vista o lugar central que a Evolução ocupa na Biologia (Bizzo \& El-Hani 2009), mas se agrava quando pensamos na realidade escolar, em que os professores raramente chegam ao final do livro (Gould 1983b). A prática de colocar Evolução no final do livro ou curso deve-se ao fato de autores e professores considerarem que, virtualmente, todo o resto da Biologia é pré-requisito para o ensino de Evolução (Bizzo \& El-Hani 2009); ou, como relatado por Skoog (1984) de um modo mais sombrio, deixar a Evolução para o final torna mais conveniente para o professor omitir o tema polêmico de suas aulas.

Felizmente, essa prática tem se tornado obsoleta e a Evolução é agora sempre um dos tópicos iniciais dos livros (Swarts et al. 1994). Felizmente, os livros analisados aqui seguem a tendência atual. Entretanto, um único livro dentre esses ainda traz a Evolução como o último tópico: Trivellato et al. (2008).

\section{A questão da linguagem}

Quando definimos o "grau de erro" dos livros para a análise, atentar-se à linguagem utilizada é tão importante quanto evitar erros conceituais. Os conceitos de Evolução biológica, seja no ensino ou na mídia, têm sido sempre acompanhados de expressões errôneas (Bizzo 1994), como: "mamíferos são mais evoluídos que insetos", "o homem descende do macaco", "os dinossauros estão extintos, pois não eram evoluídos o suficiente" etc. Tais expressões vêm da ideia de progresso da scala naturae e do modo como se costuma fazer a Seleção Natural soar "Lamarckista" ou finalista (Bloom 1989; Bizzo 1994; Moore et al. 2002). Isto não é restrito somente ao texto, como mostrado por Catley et al. (2010), o uso de figuras e diagramas também tem uma influência grande em passar esse tipo de ideia errônea.

Diversos livros analisados aqui apresentam esses erros de duas maneiras: (1) Erros conceituais de fato, em que os autores parecem manter essas ideias como verdadeiras, como Trivellato et al. (2008), Stem (2009) e, em parte, Santana \& Mozena (2012). (2) Erros de linguagem simplificada, em que os autores tentaram simplificar demais o conteúdo para uma audiência mais nova, recaindo assim em erros que poderiam ser evitados de outro modo, como Bröckelmann (2008), Canto (2009b), Santana (2012) e, em parte, Santana \& Mozena (2012). Este 
segundo caso é agravado pelo uso de figuras de linguagem e analogias vagas ou enganosas (Bizzo 1994; Moore et al. 2002).

Temos em vista que, em se tratando do Ensino Fundamental II, a linguagem deve ser mais simples, mas isso não pode servir de desculpa para se repassar informações erradas aos alunos ou para "emburrecer" o conteúdo (“dumbing down” sensu Gould 1995). O conteúdo pode ser apresentado com uma linguagem simples e de forma correta. Isto é claramente possível, como demonstrado por alguns dos livros analisados aqui: Canto (2009a), Godoy \& Ogo (2012) e Usberco et al. (2012).

\section{Os erros conceituais}

Muitos dos erros conceituais do item (1) do tópico acima podem ser devido ao fato de que livros didáticos tendem a ser cópias de cópias, cujos textos (e erros) ficam "fossilizados"; Gould (1991) apresentou bem esse problema. Contudo, erros conceituais podem sim ser falhas na formação dos autores, que supostamente são os melhores dentre os professores, como argumentado por Jiménez-Aleixandre (1994). Uma compreensão errônea da Teoria da Evolução por parte dos alunos é particularmente problemática, pois, como já ressaltamos diversas vezes, essa é a teoria fundamental da Biologia (Moody 1996).

Assim, não podemos evitar em falar na capacitação continuada dos professores. Os professores de Ciências e Biologia foram objeto de estudo em diversas pesquisas, onde procurouse por fatores que constituíssem obstáculos no ensino da Teoria da Evolução para seus alunos. Tais pesquisas identificaram professores com erros conceituais (Rutledge \& Warden 2000; De Assis et al. 2008; Smith 2010b; Yates \& Marek 2013), conflitos ideológicos (Bloom 1989; Berkman \& Plutzer 2011; Long 2012) e problemas com a abordagem pedagógica (JiménezAleixandre 1994; Smith 2010b).

Muitos professores não têm domínio sobre tópicos complexos como Evolução (por exemplo, como distinguir "Lamarckismo" de "Darwinismo", qual é a função do acaso no processo, ou ainda como de fato opera a Seleção Natural) ou o funcionamento da Ciência como um todo (Carrey \& Stauss 1970; Bloom 1989; Scharmann \& Harris 1992; Jiménez-Aleixandre 1994; Rutledge \& Mitchell 2002). Isso pode ser devido a uma falha na educação que esses professores receberam no Ensino Superior (Costa et al. 2011) ou, mais comumente, devido ao fato de que eles não são especialistas na área que ensinam, tendo formação em outras áreas (Bloom 1989; Henderson 2012). Isso é especialmente válido para os professores de “Ciências” do Ensino Fundamental (Henderson 2012). De qualquer modo, o efeito que isso tem na qualidade do trabalho desenvolvido junto aos alunos é avassalador. Algumas ideias para minimizar esses problemas são abordadas por Scharmann \& Harris (1992) e Henderson (2012).

\section{A questão do Lamarck}

O argumento de Gould (1991: 166) resume bem esta questão: "Why begin teaching evolution by explicating a false theory that is causing no confusion? False notions are often wonderful tools in pedagogy, but not when they are unknown, are provoking no trouble, and make the grasp of an accepted theory more difficult."

Apesar de ser útil ter uma perspectiva histórica das ciências (Ross 2000), livros didáticos não são o lugar para colocar todas as interpretações possíveis do mundo natural, como, por exemplo, uma Terra plana (Beard 1986). Isso é especialmente válido caso as ideias anteriores causem mais problemas do que benefícios, como é o caso de Lamarck (Gould 1991). O uso de Lamarck como um contraste à explicação Darwinista de Evolução pode até funcionar em certos casos (e.g., Jiménez-Aleixandre 1992), mas a energia dispendida nisso e a chance de gerar mais confusão são tão grandes, que não parece uma metodologia adequada (contra Almeida \& Falcão 2010).

Mesmo com um currículo mais rico do ponto de vista histórico (e incluindo longas discussões sobre Lamarck) sendo elaborado e utilizado por Jensen \& Finley (1996), foram obtidas escassas evidências de melhoria no aprendizado. Em todo caso, o "Lamarckismo" apresentado 
atualmente em livros didáticos e na mídia em geral é uma distorção grave dos trabalhos de Lamarck (Almeida \& Falcão 2005, 2010). Se o "Lamarckismo" deveria ter alguma função didática (Jiménez-Aleixandre 1992), claramente não a tem no formato atual (Almeida \& Falcão 2010). Dentre os livros analisados no presente trabalho, Canto (2009a) é o que traz menos informação sobre Lamarck e, portanto, o menos propenso a causar confusão.

\section{A questão do Criacionismo}

O ensino de Evolução sempre foi restrito pelo lobby de forças externas, especialmente em países extremamente religiosos como os Estados Unidos (Skoog 1979, 1984; Berkman \& Plutzer 2010). Infelizmente, o Brasil está, atualmente, equiparando-se aos Estados Unidos na questão do Criacionismo, como mostra, por exemplo, a recente tentativa (Projeto de Lei 8099/2014, de autoria do deputado e pastor Marco Feliciano) de tornar obrigatório o ensino do Criacionismo nas escolas. Apesar de diversas associações e sociedades brasileiras vinculadas à educação e à ciência terem se pronunciado contra, essa pressão de entidades religiosas antievolucionistas ainda é preocupante. E não só por ser uma atitude medieval em pleno século XXI, mas também pelo exposto por Gould (1983b: 282): "No arm of the industry is as cowardly and conservative as the publishers of public school texts - markets of millions are not easily ignored." É importante que as editoras não cedam às pressões dessas forças externas, ao contrário do que ocorreu nos Estados Unidos na segunda metade do século XX (Skoog 1984; Rosenthal 1985). É muito comum nos Estados Unidos que tanto os livros didáticos quanto as normas dos departamentos estaduais de educação usem linguagem ambígua e vaga para esconder, neutralizar ou enfraquecer o material referente à Evolução (Skoog \& Bilica 2002; Berkman \& Plutzer 2010; Long 2012).

Felizmente, nenhum dos livros analisados contém o disclaimer se desculpando pela Evolução que é visto nos Estados Unidos (Rosenthal 1985). Contudo, alguns livros brasileiros que não estão no PNLD contém tal disclaimer (e.g., Gewandsznajder 2012) e são adotados em escolas particulares. Infelizmente, boa parte dos livros analisados (Godoy \& Ogo 2012; Santana 2012; Santana \& Mozena 2012; Usberco et al. 2012) ainda incluem Criacionismo antes de explicar Evolução, passando a ideia de que Evolução (e, por tabela, a Ciência em si) é somente mais uma dentre as inúmeras "visões de mundo válidas".

Este último ponto está relacionado em grande parte ao fato de a Evolução, em geral, ser apresentada como "Teoria da Evolução" e o termo "teoria", na linguagem popular, estar longe de sua definição científica. Assim, o público (e os alunos) recebe a ideia de que a Evolução é "só uma teoria", como se fosse um palpite ao invés de um corpo conceitual bem estabelecido e confirmado por inúmeras linhas de evidência (Gould 1983a; Bloom 1989; Smith 2010a; Allmon 2011). Um dos modos de se corrigir isso, obviamente, é através de uma melhor educação científica. Como apontado por Smith et al. (1995: 23), "large numbers of people reject the theory of evolution, and the Science education community has done little to help teachers present evolution in a way that will ameliorate this situation."

\section{Considerações finais}

A falha mais grave da maioria dos livros didáticos analisados é não apresentar a Evolução como a teoria fundamental e unificadora da Biologia, um fato também visto em materiais didáticos de outros países. Isso impede que o aluno compreenda a teoria de modo que consiga aplicá-la para interpretar os fenômenos biológicos.

Apesar da proporção do conteúdo dos livros dedicados à Evolução (Tabela 1) ser um indicativo importante de quanta atenção é dada ao tema pelos autores, é igualmente importante saber se os autores tratam o tema de forma central para a Biologia. Dentre os livros analisados, apenas Godoy \& Ogo (2012) e Usberco et al. (2012) se aproximam disso, particularmente ao explicitar a origem e as relações evolutivas dos grandes grupos e ao explorar os fósseis; mas ainda assim, ambos poderiam deixar ainda mais explícita a função central da Evolução. 
Assim como o observado em livros do Ensino Médio (Dalapicolla et al. 2015), os livros do Ensino Fundamental apresentaram diferenças consideráveis na forma e qualidade com a qual trabalham a Teoria da Evolução, mesmo que todas as coleções avaliadas sejam aprovadas pelo PNLD. Destaca-se aqui a importância de o professor atentar à forma como a Evolução é trabalhada no livro de sua escolha, para ser possível utilizá-la como teoria unificadora em suas aulas. Além disso, o funcionamento da Ciência como um todo raramente é explicado de forma adequada e isso traz consequências negativas ao ensino de Evolução. Esta área talvez seja onde a formação do professor tem um papel mais crítico.

O presente trabalho contribui na análise do ensino de Evolução no Brasil, mas ainda há muitos outros tópicos e metodologias a serem abordados, como o desenvolvimento de um estudo semelhante para livros do Ensino Médio, a comparação entre a apresentação do tema de evolução humana (não abordada por dois dos livros analisados: Trivellato et al. 2008 e Godoy \& Ogo 2012) com a evolução de outros organismos (e.g., presença de ideias de progresso, vocabulário scala naturae, censura do tema) e o estudo de materiais paradidáticos, dado que esses são mais acessíveis para o público geral.

Finalmente, o conteúdo religioso dos livros precisa ser analisado mais a fundo para se determinar a proporção de livros (tanto os listados pelo PNLD quanto os demais) em que o Criacionismo está presente e: (1) se ele é apresentado como alternativa válida à Evolução (e, portanto, à Ciência) ou (2) se a religião é abordada somente para evitar ofender grupos de grande força política (essa tentativa de agradar gregos e troianos é vista nos Estados Unidos). Em qualquer um dos dois casos, a presença do Criacionismo em livros de Ciência é danosa e uma causa relevante do "analfabetismo" científico atual, que é especialmente alarmante no Brasil (e.g., Instituto Abramundo 2014).

\section{Agradecimentos}

Os autores são muito gratos a todas as editoras que ofereceram as coleções do PNLD para nossa análise. Somos especialmente gratos a Mariana S. Durigan, pela ajuda para conseguir algumas dessas obras e a Barbara M. Tomotani, pelos comentários e discussão sobre a metodologia do trabalho. Por fim, os autores agradecem aos dois revisores anônimos pela importante revisão crítica do manuscrito.

\section{Referências}

Allmon W.D. (2011) Why Don't People Think Evolution Is True? Implications for Teaching, In and Out of the Classroom. Evolution: Education and Outreach, 4(1): 648-665.

Almeida A.V. \& Falcão J.T.R. (2005) A estrutura histórico-conceitual dos programas de pesquisa de Darwin e Lamarck e sua transposição para o ambiente escolar. Ciência \& Educação, 11(1): $17-32$.

Almeida A.V. \& Falcão J.T.R. (2010) As teorias de Lamarck e Darwin nos livros didáticos de Biologia no Brasil. Ciência \& Educação, 16(3): 649-665.

Almeida D.F. (2012) Concepções de Alunos do Ensino Médio Sobre a Origem das Espécies. Ciência \& Educação, 18(1): 143-154.

Amaral I.A. \& Megid Neto J. (1997) Qualidade do Livro Didático de Ciências: o que define e quem define? Ciência \& Ensino, 2: 13-14.

Beard J. (1986) Comment on "Evolution in High School Biology Textbooks: 1963-1983" by Dorothy B. Rosenthal, Science Education 69(5): 637-648. 1985. Science Education, 70(5): 501502.

Berkman M.B. \& Plutzer E. (2010) Evolution, Creationism, and the Battle to Control America's Classrooms. Cambridge: Cambridge University Press. 304 p.

Berkman M.B. \& Plutzer E. (2011) Defeating Creationism in the Courtroom, But Not in the Classroom. Science, 331(6016): 404-405. 
Bizzo N.M.V. (1994) From Down House Landlord to Brazilian High School Students: What Has Happened to Evolutionary Knowledge on the Way? Journal of Research in Science Teaching, 31(5): 537-556.

Bizzo N.M.V. \& El-Hani C.N. (2009) O arranjo curricular do ensino de evolução e as relações entre os trabalhos de Charles Darwin e Gregor Mendel. Filosofia e História da Biologia, 4: 235-257.

Bloom J.W. (1989) Preservice elementary teachers' conceptions of science: science, theories and evolution. International Journal of Science Education, 11(4): 401-415.

Borges R.M.R. \& Lima V.M.R. (2007) Tendências contemporâneas no ensino de Biologia no Brasil. Revista Electrónica de Enseñanza de las Ciencias, 6(1): 165-175.

BRASIL. MEC/SEB. (2013) Guia de livros didáticos: PNLD 2014: ciências: ensino fundamental: anos finais. Brasília: SEB/FNDE. 40 p.

Bröckelmann R.H. (2008) Observatório de Ciências 7. São Paulo: Moderna. 272 p.

Canto E.L. (2009a) Ciências Naturais. Aprendendo com o Cotidiano. $7^{\circ}$ ano. $3^{\circ}$ edição. São Paulo: Moderna. $272 \mathrm{p}$.

Canto E.L. (2009b) Ciências Naturais. Aprendendo com o Cotidiano. $9^{\circ}$ ano. $3^{\circ}$ edição. São Paulo: Moderna. $215 \mathrm{p}$.

Carey R.L. \& Stauss N.G. (1970) An analysis of experienced science teachers' understanding of the nature of science. School Science and Mathematics, 70(5): 366-376.

Catley K.M., Novick L.R. \& Shade C.K. (2010) Interpreting evolutionary diagrams: when topology and process conflict. Journal of Research in Science Teaching, 47(7): 861-882.

Costa L.O., Melo P.L.C. \& Teixeira F.M. (2011) Reflexões acerca das diferentes visões de alunos do Ensino Médio sobre a origem da diversidade biológica. Ciência \& Educação, 17(1): 115-128.

Dalapicolla J., Silva V.A. \& Garcia J.F.M.G. (2015) Evolução biológica como eixo integrador da biologia em livros didáticos do ensino médio. Ensaio Pesquisa em Educação em Ciências, 17(1): 150-172.

De Assis J.E., Christoffersen M.L., Alonso C. \& Araújo-De-Almeida E. (2008) Avaliando o conceito de evolução biológica dos professores de Biologia do Ensino Médio de João Pessoa, Paraíba, Brasil. Gaia Scientia, 2(1): 97-105.

Department for Education (2015) National Curriculum in England: Science Programmes of Study. Disponível em: https://www.gov.uk/government/publications/national-curriculum-inengland-science-programmes-of-study (Acessado em 24.07.2015).

Dobzhansky T. (1973) Nothing in Biology Makes Sense except in the Light of Evolution. The American Biology Teacher, 35(3): 125-129.

Freire P. (1996) Pedagogia da Autonomia: Saberes Necessários à Prática Educativa. Rio de Janeiro: Paz e Terra. $92 \mathrm{p}$.

Gewandsznajder F. (2012) Ciências. Vida na Terra. Projeto Teláris. São Paulo: Ática. 352 p.

Godoy L.P. \& Ogo M.Y. (2012) Vontade de Saber Ciências 7. São Paulo: FTD. 306 p.

Goldston M.J. \& Kyzer P. (2009) Teaching evolution: narratives with a view from three southern biology teachers in the USA. Journal of Research in Science Teaching, 46(7): 762-790.

Good R. (1992) Evolution education: an area of needed research. Journal of Research in Science Teaching, 29(10): 1019.

Gould S.J. (1983a) Evolution as a fact and theory (p. 253-262). In: Gould S.J. Hen's Teeth and Horse's Toes. Further Reflections in Natural History. W.W. New York: Norton \& Company. $416 \mathrm{p}$.

Gould S.J. (1983b) Moon, Mann and Otto (p. 280-290). In: Gould S.J. Hen’s Teeth and Horse's Toes. Further Reflections in Natural History. W.W. New York: Norton \& Company. 416 p.

Gould S.J. (1991) The case of the creeping fox terrier clone (p. 155-167). In: Gould S.J. Bully for Brontosaurus. W.W. New York: Norton \& Company. 544 p.

Gould S.J. (1995) Dinosaur in a Haystack: Reflections in Natural History. New York: Harmony Books. 409 p.

Henderson M. (2012) The Geek Manifesto: Why Science Matters. London: Bantam Press. 416 p. 
Hokayem H. \& Boujaoude S. (2008) College students' perceptions of the theory of evolution. Journal of Research in Science Teaching, 45(4): 395-419.

IBOPE. (2004) Pesquisa de opinião pública sobre o criacionismo, 2004. Disponível em: http://www.ibope.com.br/pt-br/conhecimento/relatoriospesquisas/lists/relatoriospesquisaeleitor al/opp\%20040992\%20-\%20criacionismo.pdf. (Acessado em 23.07.2015).

Instituto Abramundo (2014). Indicador de Letramento Científico. Relatório Técnico da Edição 2014. Disponível em: http://cienciahoje.uol.com.br/noticias/2014/08/imagens/IndiceLetramento-Cientifico.pdf. (Acessado em 26.07.2015).

Jeffery, K.R. \& Roach L.E. (1994) A study of the presence of evolutionary protoconcepts in prehigh school textbooks. Journal of Research in Science Teaching, 31(5): 507-518.

Jensen M.S. \& Finley F.N. (1996) Changes in students' understanding of evolution resulting from different curricular and instructional strategies. Journal of Research in Science Teaching, 33(8): 879-900.

Jiménez-Aleixandre M.P. (1992) Thinking about theories or thinking with theories?: a classroom study with natural selection. International Journal of Science Education, 14(1): 51-61.

Jiménez-Aleixandre M.P. (1994) Teaching evolution and natural selection: a look at textbooks and teachers. Journal of Research in Science Teaching, 31(5): 519-535.

Krippendorff K. (2013) Content analysis: An introduction to its methodology. $3^{\circ}$ edition. Los Angeles: Sage. 456 p.

Levin F.S. \& Lindbeck J.S. (1979) An analysis of selected biology textbooks for the treatment of controversial issues and biosocial problems. Journal of Research in Science Teaching, 16(3): 199-203.

Long D.E. (2012) The Politics of Teaching Evolution, Science Education Standards, and Being a Creationist. Journal of Research in Science Teaching, 49(1): 122-139.

Maclure S. (1986) Educational Documents: England and Wales, 1816 to the Present Day. $5^{\circ}$ edition. London: Methuen. 448 p.

Martins, I. (2006) Analisando livros didáticos na perspectiva dos Estudos do Discurso: compartilhando reflexões e sugerindo uma agenda para a pesquisa. Pro-Posições, 17(1): 117136.

Mazur A. (2005) Believers and disbelievers in evolution. Politics and Life Sciences, 23(2): 55-61.

Megid Neto J. \& Fracalanza H. (2003) O Livro Didático de Ciências: Problemas e Soluções. Ciência \& Educação, 9(2): 147-157.

Millar R. \& Osborne J. (1998) Beyond 2000: Science education for the future. London: King's College. $32 \mathrm{p}$.

Miller J.D., Scott E.C. \& Okamoto S. (2006) Public Acceptance of Evolution. Science, 313(5788): 765-766.

Moody D.E. (1996) Evolution and the Textbook Structure of Biology. Science Education, 80(4): 395-418.

Moore R., Mitchell G., Bally R., Inglis M., Day J. \& Jacobs D. (2002) Undergraduates' understanding of evolution: ascriptions of agency as a problem for student learning. Journal of Biological Education, 36(2): 65-71.

Oliveira G.S. \& Bizzo N. (2011) Aceitação da evolução biológica: atitudes de estudantes do ensino médio de duas regiões brasileiras. Revista Brasileira de Pesquisa em Educação em Ciências, 11(1): 57-79.

Penteado P.R., Kavalco K.F. \& PAZZA, R. (2012) Influence of sociocultural factors and acceptance of Creationism in the comprehension of evolutionary biology in freshman Brazilian students. Evolution: Education and Outreach, 5: 589-594.

Rist R.C. (1982) On the application of ethnographic inquiry to education: procedures and possibilities. Journal of Research in Science Teaching, 19(6): 439-450.

Rosenthal D.B. (1985) Evolution in High School Biology Textbooks: 1963-1983. Science Education, 69(5): 637-648.

Ross A. (2000) Curriculum. Construction and Critique. London: Falmer Press. 204 p. 
Rutledge M.L. \& Mitchell M.A. (2002) High School Biology teachers' knowledge structure, acceptance \& teaching of evolution. The American Biology Teacher, 64(1): 21-28.

Rutledge M.L. \& Warden M.A. (2000) Evolutionary Theory, the Nature of Science \& High School Biology Teachers: Critical Relationships. The American Biology Teacher, 62(1): 23-32.

Santana O.A. (2012) Ciências Naturais. $7^{\circ}$ ano. São Paulo: Saraiva. 320 p.

Santana O.A. \& Mozena E. (2012) Ciências Naturais. $9^{\circ}$ ano. São Paulo: Saraiva. 336 p.

Scharmann L.C. \& Harris W.M. (1992) Teaching evolution: understanding and applying the nature of Science. Journal of Research in Science Teaching, 29(4): 375-388.

Sinatra G.M., Southerland S.A., Mcconaughy F. \& Demastes J.W. (2003) Intentions and beliefs in students' understanding and acceptance of biological evolution. Journal of Research in Science Teaching, 40(5): 510-528.

Skoog G. (1979) Topics of Evolution in Secondary School Biology Textbooks: 1900-1977. Science Education, 63(5): 621-640.

Skoog G. (1984) The Coverage of Evolution in High School Biology Textbooks Published in the 1980s. Science Education, 68(2): 117-128.

Skoog G. (2005) The Coverage of Human Evolution in High School Biology Textbooks in the 20th Century and in Current State Science Standards. Science \& Education, 14(3-5): 395-422.

Skoog G. \& Bilica K. (2002) The emphasis given to evolution in state Science standards: a lever for change in evolution education? Science Education, 86(4): 445-462.

Smith M.U. (2010a) Current Status of Research in Teaching and Learning Evolution: I. Philosophical/Epistemological Issues. Science \& Education, 19(6-8): 523-538.

Smith M.U. (2010b) Current Status of Research in Teaching and Learning Evolution: II. Pedagogical Issues. Science \& Education, 19(6-8): 539-571.

Smith M.U., Siegel H. \& Mcinerney J.D. (1995) Foundational issues in evolution education. Science \& Education, 4(1): 23-46.

Stern I. (2009) Ciências no Século XXI. Seres Vivos. $7^{0}$ ano / $6^{\mathrm{a}}$ série. São Paulo: Atual. 295 p.

Swarts F.A., Anderson O.R. \& Swetz F.J. (1994) Evolution in Secondary School Biology Textbooks of the PRC, the USA, and the Latter Stages of the USSR. Journal of Research in Science Teaching, 31(5): 475-505.

Trivellato J., Trivellato S., Motokane M., Lisboa J.F. \& Kantor C. (2008) Ciências. Natureza \& Cotidiano. Criatividade, Pesquisa, Conhecimento. $7^{\circ}$ ano $/ 6^{\mathrm{a}}$ série. São Paulo: FTD. 234 p.

Usberco J., Martins J.M., Schechtmann E., Ferrer L.C. \& Velloso H.M. (2012) Companhia das Ciências 7. São Paulo: Saraiva. 277 p.

Yates T.B. \& Marek E.A. (2013) Is Oklahoma really OK? A regional study of the prevalence of biological evolution-related misconceptions held by introductory biology teachers. Evolution: Education and Outreach, 6(1): 1-20.

Zimmerman M. (1987) The evolution-creation controversy: Opinions of Ohio high school biology teachers. Ohio Journal of Science, 87(4): 115-125. 